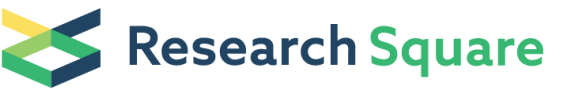

\section{Intermittent fasting induces chronic changes in the hepatic gene expression of Red Junglefowl (Gallus gallus)}

Caroline Lindholm ( $\nabla$ caroline.lindholm@liu.se )

Linkopings universitet https://orcid.org/0000-0001-8961-3193

\section{Petros Batakis}

Linkopings universitet

Jordi Altimiras

Linkopings universitet

John Lees

Linkopings universitet

Research article

Keywords: Gallus gallus, intermittent fasting, liver transcriptomics, microarray, red junglefowl, skip-a-day

Posted Date: September 24th, 2019

DOI: https://doi.org/10.21203/rs.2.14882/v1

License: @ (i) This work is licensed under a Creative Commons Attribution 4.0 International License.

Read Full License 


\section{Abstract}

Background: Intermittent fasting, the implementation of fasting periods of at least 12 consecutive hours on a daily to weekly basis, has received a lot of attention in recent years for imparting the life-prolonging and health-promoting effects of caloric restriction with no or only moderate actual restriction of caloric intake. Intermittent fasting is also widely practiced in the rearing of so-called broiler breeders, the parent stock of meat-type chickens, who require strict feed restriction regimens to prevent the serious health problems associated with their voracious appetites. Although intermittent fasting has been extensively used in this context to reduce feed competition and its resulting stress it has not usually been considered as a health-span promoter, but presents an alternative and complementary model to rodent studies. In both mammals and birds, the liver is one of the main responders to variations in energy balance. In this paper we examine the liver transcriptomics of wild-type Red Junglefowl chickens fed either ad libitum, chronically restricted to around $70 \%$ of ad libitum daily or intermittently fasted on a 2:1 (2 days fed, 1 day fasted) schedule without actual caloric restriction using a microarray.

Results: We find large effects of feeding regimen on liver transcriptomics, with most of the affected genes relating to energy metabolism. Interestingly we also find large, chronic effects on the transcription of a battery of cell proliferation-promoting genes. Similar regenerative effects have been reported in rodents, where they have been associated with reduced levels of circulating leptin. A small number of inflammation-related genes also show chronically changed expression profiles.

Conclusions: The increase in proliferative potential suggested by the gene expression changes reported here is a first indication that birds and mammals respond similarly to intermittent fasting practices. Our findings may indicate that the health benefits of periodic caloric restriction are ubiquitous and note restricted to mammals alone. The mechanisms underpinning these benefits are, however, unclear as leptin has been associated with these benefits in mammals but does not have endocrine function in birds. The observed effects on inflammation-related genes may be either beneficial or detrimental for the animal and could warrant further investigation.

\section{Background}

Intermittent fasting (IF) has gained a lot of attention in recent years for imparting many of the health benefits associated with caloric restriction, without actually restricting total caloric intake (1). IF is typically defined as eating patterns involving at least $12 \mathrm{~h}$ of continuous fasting on a daily to weekly basis, although regimens including minimal consumption ( $<25 \%$ of baseline metabolic needs) in place of fasting or $8 \mathrm{~h}$ daily fasts are sometimes also included (2). Reported health benefits of IF include weight loss with maintained lean mass (3), improved insulin sensitivity (3) and protection from age-related (4) as well as pathology-induced cognitive decline (5). Many of the health benefits associated with IF have been attributed to the metabolic switching between a glucose-based and a ketone-based energy metabolism that comes about as a result of hepatic glycogen stores being depleted within $12-36 \mathrm{~h}$ after the last consumed meal (2). This switch is driven by metabolic processes in the liver, where energy originally 
supplied through glycogenolysis is replaced by that from ketogenesis and gluconeogenesis, and will eventually induce changes in energy substrate preference in peripheral tissues $(2,6)$.

Models of IF in mammals have risen in popularity in recent years but IF is also well established in the rearing of meat-type chickens, often under the name "skip-a-day" feeding (7). Feed restriction is routinely used in the rearing and maintenance of so called "broiler breeders", the meat-type parent stock, to combat long-term health problems associated with the extreme appetites that these animals are bred for $(7,8)$. IF regimens are commonly used in this setting to increase portion sizes and to reduce food competition and the resulting meal-time stress and aggression $(7,8)$. Despite this, IF in farmed birds is widely considered detrimental to animal health and welfare (7) and has even been linked to increased adiposity in some studies $(9,10)$.

One reason for the seemingly opposing views on IF in the mammalian vs poultry literature may be that meat-type chickens cannot be fed ad libitum (AL) long-term without developing severe health problems and excessive mortality $(11,12)$ and are thus usually restricted to around $30 \%$ of ad libitum intake even in IF studies. In this paper, we aim to better understand the response to IF in poultry by instead using the Red Junglefowl (RJF), a wild-type chicken which is not prone to diet-induced obesity and remains healthy on an $A L$ diet. We raised a cohort of young RJF on either AL feeding, chronic daily restriction (CR) to around $70 \%$ of AL intake or an IF diet (two feeding days at $150 \%$ of daily AL intake offered followed by a fasting day) from 2-5 weeks of age. Birds fed according to the CR and IF feeding regimens showed similar growth rates, although IF-fed birds had a consistently higher feed intake as previously reported (13). It is well known that the liver is central to the primary metabolic response to dietary changes in chickens (14), and shows large fluctuations in mass, glycogen content and lipid content under IF conditions in both RJF (13) and broiler breeders $(10,15)$. Concomitant with these overt physiological effects are large effects on hepatic transcription. Others have previously looked at the transcriptomic response to acute fasting in chicken liver (16) or the transcription of a subset of hepatic genes in long-term IF (15), but we are not aware of any other study that has examined the transcriptomic response to long-term IF in chickens. The intermittently fasted chickens were sampled at two different time points: the second consecutive day of feeding (F2) and the fasting day (SK), which were expected to represent the two extremes of the metabolic switch. In this study, we perform cluster and pathway analyses on the liver transcriptome at these timepoints and compare them to both liver physiology traits (liver mass, lipid content, glycogen content) and appetite-associated gene expression in the arcuate nucleus ( $\mathrm{ArcN}$ ). We also take a special interest in two types of expression patterns in IF chickens: 1) cyclically switching expression patterns where F2 and SK expression levels are significantly different as these may be associated with metabolic switching and its health benefits or drawbacks, and 2) chronic changes in gene expression where F2 and SK expression are significantly different from the AL-fed controls in a consistent direction which are potentially related to long-term health effects of IF.

\section{Results}


The flow of microarray data analysis is presented in Fig 1. In brief, data from 61,818 microarray probes were matched to 20,771 genes. Of these, 3,007 genes met the criteria of $P \leq 0.01$ and a fold change $\geq$ 1.4 , the latter chosen for comparability with previously published chicken liver transcriptomics (16). The number of up- and down-regulated genes were roughly equal in all comparisons, ranging from 124 upand 157 down-regulated genes (comparing AL and F2), to 798 up- and 786 down-regulated (comparing F2 and SK) (Table 2).. In total, 1,675 genes showed upregulation and 1,630 showed downregulation in at least one treatment comparison with some genes showing both patterns depending on which groups were being compared (Additional figure 1)..

\section{Cluster and pathway analyses}

The gene set underwent a cluster analysis, sorting genes into four clusters (Fig 2, heatmap rows). The clustering analysis successfully differentiated between the four feeding treatments, grouping the two fully fed states (AL and F2) together and the underfed states of chronic restriction and fasting (CR and SK) together. The gene clusters were also split into two cluster pairs, with clusters 1 and 2 (634 and 912 genes respectively) showing general down-regulation in the fully fed states. Cluster 1 was upregulated in CR but not in fasting, with cluster 2 showing a near-opposite pattern. Clusters 3 and 4 (501 and 960 genes respectively) instead showed general up-regulation in the fully fed states and were mostly downregulated in CR. Cluster 4 was also down-regulated in SK, but cluster 3 was not. (Fig 2).. Correlational analyses were performed to compare the mean expression of each cluster to known parameters of liver physiology and ArcN expression of appetite-related genes (Fig 3, Additional figure 2).. Clusters 1 and 3 were both significantly correlated with relative liver mass (expressed as \% body weight), and the ArcN expression of the appetitive AgRP and the anorexic POMC (Fig 3).. The correlational patterns were opposite with higher Cluster 1 expression in individuals with low relative liver mass, low POMC expression and high AgRP expression and the reversed situation for Cluster 3. Clusters 2 and 4 were instead correlated with hepatic glycogen reserves and $\mathrm{ArcN}$ expression of the consummatory orexigenic signal NPY. Again, these two clusters showed opposite patterns with Cluster 2 expression showing negative correlation with glycogen concentration and positive correlation with NPY expression, and Cluster 4 the reverse (Fig 3)..

Each cluster was also subjected to a KEGG analysis. Approximately $40 \%$ of the cluster genes were covered by the KEGG database and about half of those were included in the pathway analysis (Fig 1).. Clusters 1, 2 and 4 were heavily loaded with metabolic pathways, with the general "Metabolic pathways" category making up $36.1 \%, 27.0 \%$ and $27.0 \%$ of the transcripts respectively (Fig 4 ).. Of these, $40.6 \%$, $28.3 \%$ and $30.7 \%$ respectively were exclusive to that category. Cluster 1 mainly consists of genes related to amino acid metabolism, cluster 2 is heavily loaded with lipid and fatty acid metabolism genes and cluster 4 is home to most transcripts related to the metabolism of sugars and starch. Cluster 3 , which was by far the smallest cluster in the pathway analysis, was instead made up of genes related to DNA replication and cell cycle progression (Fig 4).. While many of the main terms highlighted in the pathway analysis are ambiguous and largely overlapping, some particularly interesting pathway terms worth noting include cell cycle (30 genes), PPAR signaling (17 genes) and insulin resistance (11 genes). Less 
relevant pathway terms include "biosynthesis of antibiotics", a set of 75 genes that entirely overlap with other pathways and are largely made up of genes involved in basic metabolic processes and "Herpes simplex infection" which contains a mostly unique collection of 15 genes involved in apoptosis (FADD, CASP8), inflammatory JAK/STAT signaling (SOCS3, JAK1, IFNGR2), transcriptional activation (TBPL1, ALYREF), splicing (SRSF2, SRSF3, LOC100859609) and protein synthesis (EIF2S1) as well as ERK signaling (FOS, JUN) an S-phase protein (SKP2) and a circadian regulator (CLOCK).

\section{Expression pattern profiling}

In addition to the cluster-based analysis we performed a pattern analysis looking for genes with expression profiles of particular interest. The same 3,007 genes that were subjected to the cluster and KEGG pathway analyses were also examined for expression patterns that could be of certain interest in the context of metabolic switching. For this reason we looked for two different expression patterns: either a pattern directly resembling the metabolic switch with F2 and SK being significantly different from each other or a chronic change in expression (F2 and SK both significantly different from AL and changed in the same direction) which would be expected for mechanisms involved in long-term health effects of metabolic switching.

A total of 967 genes met the switching criteria with the expression of at least one of the IF days being significantly different from AL, although this includes some duplicates due to retired annotations (Fig 1).. For further analysis, we chose to restrict ourselves to the 27 genes that were significantly different from AL under both F2 and SK conditions. Of these, seven entries also met the criteria for chronic change and will be considered as part of that dataset. A total of 13 genes were found to be unique, identifiable and have mammalian orthologs that have been characterized; one more was identifiable but uncharacterized (TCP11L2) and four were unidentifiable. The expression profiles of these genes are presented in Fig 5. Six out of the 13 characterized genes show a switching pattern where expression is significantly lower in F2 conditions but elevated during fasting (Fig 5a),, of these five are mainly related to energy metabolism (TMEM234, HAO2, CMBL, MMADHC, GYS2) and one is mainly related to immune function (F11). These genes were typically unaffected by the CR treatment. The remaining seven genes (Fig $5 b-c$ ) showed the opposite pattern of elevated expression during feeding but reduced expression in fasting, of these two are mainly implicated in cell proliferation (ODC1, DUSP14), one is immune-related (IRF9; two transcripts) and one mainly involved in energy metabolism (ITPR3). The remaining three are involved in telomere elongation (SMG6), membrane transport (XKRX), and tubulin formation (TUBB2B). Most of these genes showed some level of reduced expression in CR compared to AL. A brief overview of the function of these genes is given in Table 2.

The chronic change criteria were met by a total of 70 genes, of which 49 were unique, identifiable and characterized (Fig 1).. Six of these correspond to the seven entries (i.e. one gene was represented by two transcripts) also showing a switching pattern, and the remaining 43 genes were non-switching. Five of the six genes showing both chronic change and a switching pattern were chronically upregulated compared to AL (Fig 6a), , four of these are mainly related to energy metabolism (HMGCL, SUCNR1, 
NR1D2 (two transcripts) and AGPAT9) and one mainly to cell proliferation (PLZF). All of these showed a switching pattern with higher expression in fasting conditions, while the only gene that was downregulated compared to AL (MTHFR, Fig 6b) was also further down-regulated in SK. The function of these genes is briefly summarized in Table 3 . The remaining 43 genes were fairly evenly split into 21 characterized (Fig 7a-d) and five uncharacterized genes (Fig 7e) that were upregulated and 22 characterized (Fig $7 f-i)$ and six uncharacterized genes (Fig $7 i-j)$ that were down-regulated compared to AL controls. The upregulated gene set was made up of 13 cell proliferation genes (HTATIP2, PAICS, HISTH1, BIRC5, CDHR2, KIF20A, NHEJ1, FANCI, BRCA2, HIST1H3H (two transcripts), HISTH110, CIP2A, CA9), two energy metabolism genes (ACAT2, CHPT1), one circadian gene (PER3), one immune-related gene (AvBD13; two transcripts), one monoamine oxidase (MAOA), one adhesion molecule (CD99L2), one potential cytoskeletal effector (PYROXD2) and one negative growth factor (CG-16). The downregulated gene set was made up of five energy metabolism genes (SLCO1B3, MYLIP, KCNT2, LPL, HRASLS, UGT1A1), three cell proliferation genes (JARID2, SDC1, RALGPS1), three immune-related genes (CFH, C4A, C4BPA), two circadian genes (DDX5, TIPARP), two transport proteins (SLC40A1, SLC10A7), one Golgi protein (FAM198B), one cell motility gene (SPATA4), two deaminating proteins (ABHD12B, ACCS), one mitochondrial metabolism gene (YME1L1) and one antioxidant response transcription factor (NFE2L2, two transcripts). The functions of these genes are briefly described in Table 4.

Of the 49 genes whose expression levels were chronically affected by IF, ten were chronically changed compared to both $\mathrm{AL}$ and $\mathrm{CR}$ (Fig 7a-e, i).. The expression of each of these genes were correlated against the same traits of liver physiology and appetite regulation expression in the $\mathrm{ArcN}$ as in the cluster analysis (Fig 8).. The expression of HTATIP2, CDHR2, KIF20A, HISTH3H, CIP2A, CG-16, NFE2L2 and BIRC5 were significantly correlated with the relative mass of the liver (expressed as \%BW) with only the last two mentioned showing negative correlations. The expression of CIP2A was also significantly and positively correlated with the hepatic total lipid concentration. No other significant correlations were found.

\section{Discussion}

This study is the first to report large-scale transcriptomics of the liver's response to long-term IF in chickens. In a similar study using an acute one-time fasting challenge changes in hepatic gene expression consisted mostly of down-regulation in the fasting birds (16). In this study, however, we find similar numbers of up- and down-regulated genes across treatments which may reflect that the birds had acclimated to their feeding treatments and did not perceive them as an acute challenge. While we have focused solely on the liver in this study, our expectation is that the liver represents the bulk of the transcriptomic changes experienced under nutritional challenges. It has been previously shown that different diets lead to changed expression patterns in the liver, but not in muscle or adipose tissue (14). Most of the genes whose expression levels were altered by different diet composition (high vs low fat) in that study appear in Clusters 3 and 4 in the current study, with only two appearing in Cluster 2 (CPT1A, LPIN1). Only one member of their "fatty acid metabolism transcription factors" was differentially expressed in our study (JUN), appearing in Cluster 4. 
As expected, most of the observed differences in gene expression in the current study are related to energy metabolism, with Clusters 2 and 4 especially showing the switching pattern between fed and fasted days in the IF group. The KEGG pathway analysis identified these clusters as largely containing lipid vs carbohydrate metabolism genes, which goes well with the physiological processes known to be involved in metabolic switching and fasting. Cluster 1 was identified from the pathway analysis as largely amino acid metabolism and also shows some switching, but mainly appears to differentiate the $\mathrm{CR}$ birds from the other groups. What stands out as more interesting are the wide-spread effects on cell cycle transcriptomics, which are mostly clustered into Cluster 3 . The expression patterns of Cluster 3 genes generally suggest increased hepatocyte proliferation in IF chickens while showing reduced expression in the growth-matched CR group. Transcriptomic signs of increased cell proliferation potential have previously been reported in the liver of chickens experiencing a one-time $48 \mathrm{~h}$ fasting challenge (16) and were then described as surprising. Our results suggest that this is indeed an important response to both long- and short-term fasting. Although these effects may initially seem surprising, they agree with what has been reported in rodents. For example, acute fasting for $48 \mathrm{~h}$ in rats has been shown to lead to increased cell proliferation rates after refeeding (17) and mice fed an IF diet showed increased neural proliferation after sham surgery (18). In the mice this effect was linked to lower levels of circulating leptin, however this association appears unlikely in chickens where leptin does not appear to have an endocrine function (19) and may suggest that a more conserved mechanism is likely to be driving the effects on proliferation.

Our expression pattern analysis highlighted 10 genes as particularly important as their expression levels were chronically changed in IF (ie. in both F2 and SK) compared to both AL and CR conditions. In general the expression of these genes showed no or poor correlation with the liver physiology traits and $\mathrm{ArCN}$ appetite regulatory expression tested, suggesting that they add physiological information that the other traits we have recorded do not reflect. Of the 10 genes, six are related to cell proliferation namely HTATIP2, BIRC5 (isoform survivin 3), CDHR2, KIF20A, HIST1H3H and CIP2A. All six of these genes showed chronic upregulation in IF compared to AL and CR. Protein expression of HTATIP2 is known to be correlated with increases in triglyceride accumulation and decreases in fatty acid oxidation in mice (20). This gene appears to be key to down-regulate glycolysis and oxygen consumption in glucose-limited conditions (21), and also functions as a redox sensor that induces apoptosis in response to oxidative stress (22). While HTATIP2 can promote apoptosis, BIRC5 is an apoptosis inhibitor that promotes cell cycle progression in the liver (23). Liver-specific knockout of BIRC5 reduces the regenerative capacity in mouse liver after surgery (24), seemingly opposite to the effects of IF reported by Manzanero et al (18). KIF20A, HIST1H3H and CIP2A are all vital to cell cycle progression with KIF20A being involved in mitotic abscission (25), HIST1 H3H being a replication-dependent histone specifically expressed during $S$ phase (26) and CIP2A knockdown known to significantly increase the proportion of non-cycling cells (27). In slight contrast to the other proliferation-related genes, CDHR2 is a contact inhibition trigger, leading to reduced proliferation rates when cell confluency is high $(28,29)$. In total, the increased expression of these six genes should lead to a higher plasticity of IF livers, allowing them to quickly adapt to varying availability of energy substrates in general and glucose especially. This kind of improved proliferative 
control also appears to be the case in mice on IF feeding schedules which show reduced cellular aging in the liver with reductions in both proliferation and apoptosis suggesting improved cellular maintenance rather than increased turnover of cells (30), and lower levels of neural cell death with less dysfunctional neurogenesis after a stroke (18).

Of the remaining chronically affected genes, three may be related to inflammatory processes. The only upregulated gene among them is $\mathrm{CG}-16$, a chicken galectin that uniformly binds to chicken lymphocytes and has been shown to reduce the T cell response by $41-55 \%$ (31). In mammals, galectins are known to induce $T$ cell apoptosis and are important for down-regulation of the immune response after inflammation (32). NFE2L2 can also decrease inflammation and acts as a transcription factor to protect cells from oxidative damage (33) but was chronically down-regulated in the IF birds in this study. Increased oxidative damage has been previously reported in conjunction with increased respiratory capacity in the liver of IF-fed rats suggesting that this gene may warrant further attention (34). The opposite pattern of increased NFE2L2 expression has previously been reported in broiler chickens experiencing cold stress (35) or fed high levels of poly-unsaturated fatty acids (36). The expression of LYSMD2 was also downregulated in IF birds compared to both AL and CR. Although the actual function of the gene is yet unknown, it appears to be related to myeloid cell-mediated inflammation in humans (37) and has been found to be overexpressed in mice with fatty liver-induced hepatocellular carcinomas (38). In dogs undergoing surgery for congenital portosystemic shunts, low hepatic expression of NFE2L2 during surgery is a predictor of poor recovery (39). The expression of these three inflammation-associated genes could indicate either a reduced need or a reduced capacity for inflammatory responses in IF-fed birds. At this point we cannot say which of these two explanations is more likely, but it is worth noting that IF leads to a modest increase in heterophil-to-lymphocyte ratios (heterophils are the avian equivalent of neutrophils in mammals) which does suggest an immune activation under these conditions (10).

Finally, the expression of NR1D2 was chronically upregulated in IF livers compared to both AL and CR treatments. This gene links circadian rhythm to hepatic lipid metabolism and may also have effects on cell proliferation. NR1D2 encodes one of the two Rev-erb protein subtypes which achieve the circadian patterns in protein and lipid metabolism (40). The two subtypes are almost entirely overlapping in function which is typical for clock genes (40). Rev-erb deficiency in mice is associated with mild hypoglycemia and elevated levels of free fatty acids after a $12 \mathrm{~h}$ fast with minimal change in circulating ketones (40). Systemic double knockout mice show fragmented activity throughout the day (41). NR1D2 is also overexpressed in some cancers, where it is important for focal adhesion formation. Knockdown of NR1D2 in these cells reduced cell size and viability, suggesting effects on cell proliferation (42). The upregulation of NR1D2 in IF-fed chickens in this study may thus also contribute to a highly flexible liver that is well acclimated to the varying energy conditions typical of this feeding regimen.

\section{Conclusions}

Intermittent fasting is well studied in both chickens and rodents, but few studies have tried to answer the question of whether the health benefits of IF observed in mammals are also present in poultry. We have 
shown that the chicken liver exhibits large and chronic changes in gene expression profiles under IF regimens that do not show clear correlation with apparent liver physiology. Most interesting is a general upregulation of genes involved in cell proliferation, and we argue that this contributes to a highly flexible regulation of cell growth in the liver of IF-fed animals that potentially improves responsiveness to fluctuations in energy status. This agrees with rodent IF studies showing improved organ health and cellular maintenance.

\section{Methods}

\section{Animals management and dissections}

The Red Junglefowl used in this study came from a research population maintained at Linköping University, with its origins in Thailand (see (43) for details). The birds were raised indoors and fed ad libitum until day 14 , at which point they were switched to one of three feeding regimens as previously described in Lees et al. (13). These consisted of the following: ad libitum feeding ( $A L, n=48$ ), chronic feed restriction to first $60 \%$ (days 14-24) and then $75 \%$ (day 25 onwards) of the age-matched AL food intake corrected for body weight (CR, $n=48)$, or intermittent fasting $(I F, n=43)$ in which birds were fed $150 \%$ of their age-matched and body weight-corrected AL intake daily on two consecutive days followed by fasting on the third day. Treatments were assigned to the birds based on hatching groups (which were used as experimental groups) a priori and the individual animal was considered a statistical unit. At the end of the experimental period, birds were killed by decapitation, livers were immediately excised, weighed, frozen in liquid nitrogen and stored at $-80^{\circ} \mathrm{C}$ until the time of sample preparation. $A L$ and $C R$ birds were sampled at 36 days of age and IF birds sampled either at 40 days of age on the second consecutive day of feeding $(F 2, n=16)$ or at 41 days of age on a fasting day $(S K, n=16)$. Livers were immediately excised, weighed, frozen in liquid nitrogen and stored at $-80^{\circ} \mathrm{C}$ until the time of sample preparation. Animals were picked at random from the pen and euthanized throughout the day, individuals for the microarray were later picked for having been dissected in the 16:00-17:00 time frame while aiming for an even sex balance. Data on liver lipids and glycogen as well as ArcN gene expression have previously been published as part of a larger dataset in (13), where the assays used are also described in detail. All experimental handling of the animals, including the method of euthanasia, were approved by the Regional Council for Ethical Licensing of Animal Experiments ("Linköpings djurförsöksetiska nämnd") which is the local equivalent of an IACUC.

\section{Sample preparation and microarray analysis}

Upon thawing of samples, RNA was isolated and used to synthesize labelled cRNA for subsequent microarray analysis. Liver samples ( 30 mg) were homogenized with a FastPrep ${ }^{\circledR}-24$ (MP Biomedicals, USA) before total RNA isolation using TRIzol (Invitrogen). RNA was eluted using $30 \mu$ l of RNase-free water (Ambion, USA) and the samples analyzed for RNA quantity and quality (RNA Integrity Numbers) using a 
NanoDrop ${ }^{\circledR}$ ND-1000 (Thermo Scientific, USA), and a Bioanalyzer® instrument (Agilent Technologies, USA) respectively.

Once all RNA samples passed the quality control $(R I N \geq 8)$, they were converted to Cyanine 3-CTP labelled cRNA using one-color Low Input Quick Amp Labeling Kit (Agilent Technologies, USA), following the manufacturers' protocol. In short, 100 ng RNA per sample was mixed with 10,000-fold diluted Spike-in Mix providing a positive control for the hybridization. Samples were mixed with T7 Primer and denatured at $65^{\circ} \mathrm{C}$ for $10 \mathrm{~min}$ followed by $5 \mathrm{~min}$ incubation on ice. A master mix containing reagents for cDNA synthesis was added to all samples followed by a $2 \mathrm{~h}$ incubation at $40^{\circ} \mathrm{C}$. The enzymes were inactivated by a $15 \mathrm{~min}$ incubation at $70^{\circ} \mathrm{C}$ before addition of a transcription master mix. The samples were then incubated for $2 \mathrm{~h}$ at $40^{\circ} \mathrm{C}$ in order to synthesize complementary RNA whilst simultaneously labeling it with Cyanine 3-CTP. All samples were then purified using a RNeasy Mini Kit (QIAGEN, Germany) following the manufacturers' protocol. After NanoDrop quantification of the samples, they were hybridized to SurePrint G3 Custom 8x60K microarrays (Agilent Technologies, USA) overnight. All arrays were then scanned on an MS200 Microarray scanner (Roche NimbleGen, USA), and data was extracted via the Feature Extraction Software v12.0 (Agilent Technologies, USA). Two out of the 24 arrays failed quality control and were removed from the analysis, both from the F2 condition, leaving us with sample sizes of $\mathrm{n}=6$ for the AL, CR and SK treatments and $\mathrm{n}=4$ for the F2 treatment.

\section{Data processing}

Expression data were analysed using the Bioconductor package, limma for R. Microarray data were checked by comparing logarithmic box plots of expression signals and by performing principle component analysis of the arrays. A linear modelling approach was used to detect differentially expressed genes. To adjust for multiple testing, Benjamini-Hochberg $p$-values were applied before determining statistical significance. Genes showing a significant expression differences $(p<0.01)$ greater than 1.4-fold were included in the subsequent analysis. Clusters of genes showing similar expression patterns across the four feeding treatments were identified by two-way hierarchical cluster analysis using the hclust function in R. Probe IDs for each cluster were then converted to Ensemble gene IDs and then annotated for KEGG pathways using DAVID online Functional Annotation Tool. Overviews on gene function were based on the entries for human orthologs on genecards.org (a summary based primarily on Entrez Gene, UniProtKB/SwissGene and GeneWiki) as well as chicken-specific descriptions from UniProt were available.

\section{Declarations}

\section{Ethics approval and consent to participate}

Experimental protocols were approved by the Regional Council for Ethical Licensing of Animal Experiments (Linköpings djurförsöksetiska nämnd) and conducted under permit ID638. 


\section{Consent to publish}

Not applicable.

\section{Availability of data and material}

Microarray data has been uploaded to Array Express (http://www.ebi.ac.uk/arrayexpress) under accession E-MTAB-7829.

\section{Competing interests}

The authors declare they have no competing interests.

\section{Funding}

The study was funded by grant 2013-293 from the Swedish research council Formas to JA, LiU Neuro and the Swedish Centre of Excellence in Animal Welfare Science. JL was funded by the Carl Tryggers Stiftelse för Vetenskaplig Forskning. The funding bodies had no direct influence on any part the study or the writing of the manuscript.

\section{Authors' contributions}

JL, PB and CL set up the feeding experiment and collected tissue samples. PB performed the microarray, while $\mathrm{JL}$ and $\mathrm{CL}$ analyzed and interpreted the results and $\mathrm{CL}$ wrote the paper. All authors were involved in planning the experiment as well as read and approved the final manuscript.

\section{Acknowledgements}

The authors would like to thank Dr Dominic Wright \& Dr Martin Johnson for allowing us to use their microarray design. We would also like to thank Dr Johan Bélteky and Pia Løtvedt for invaluable help in the lab and with setting up the analysis.

\section{References}

1. Anson RM, Guo Z, de Cabo R, lyun T, Rios M, Hagepanos A, et al. Intermittent fasting dissociates beneficial effects of dietary restriction on glucose metabolism and neuronal resistance to injury from calorie intake. Proc Natl Acad Sci. 2003 May 13;100(10):6216-20.

2. Anton SD, MoehI K, Donahoo WT, Marosi K, Lee SA, Mainous AG, et al. Flipping the Metabolic Switch: Understanding and Applying the Health Benefits of Fasting: Flipping the Metabolic Switch. Obesity. 2018 Feb;26(2):254-68. 
3. Gotthardt JD, Verpeut JL, Yeomans BL, Yang JA, Yasrebi A, Roepke TA, et al. Intermittent Fasting Promotes Fat Loss With Lean Mass Retention, Increased Hypothalamic Norepinephrine Content, and Increased Neuropeptide Y Gene Expression in Diet-Induced Obese Male Mice. Endocrinology. 2016 Feb;157(2):679-91.

4. Singh R, Lakhanpal D, Kumar S, Sharma S, Kataria H, Kaur M, et al. Late-onset intermittent fasting dietary restriction as a potential intervention to retard age-associated brain function impairments in male rats. AGE. 2012 Aug;34(4):917-33.

5. Hu Y, Zhang M, Chen Y, Yang Y, Zhang J-J. Postoperative intermittent fasting prevents hippocampal oxidative stress and memory deficits in a rat model of chronic cerebral hypoperfusion. Eur $\mathrm{J}$ Nutr. 2019 Feb;58(1):423-32.

6. Cahill GF. Fuel Metabolism in Starvation. Annu Rev Nutr. 2006 Aug;26(1):1-22.

7. Morrissey KLH, Widowski T, Leeson S, Sandilands V, Arnone A, Torrey S. The effect of dietary alterations during rearing on growth, productivity, and behavior in broiler breeder females. Poult Sci. 2014 Feb 1;93(2):285-95.

8. Mench JA. Broiler breeders: feed restriction and welfare. Worlds Poult Sci J. 2002 Mar;58(1):23-9.

9. Katanbaf MN, Dunnington EA, Siegel PB. Restricted feeding in early and late-feathering chickens. 3. Organ size and carcass composition. Poult Sci. 1989 Mar;68(3):359-68.

10. Lindholm C, Johansson A, Middelkoop A, Lees JJ, Yngwe N, Berndtson E, et al. The Quest for Welfare-Friendly Feeding of Broiler Breeders: Effects of Daily vs. 5:2 Feed Restriction Schedules. Poult Sci. 2018 Feb 1;97(2):368-77.

11. Katanbaf MN, Dunnington EA, Siegel PB. Restricted feeding in early and late-feathering chickens. 1. Growth and physiological responses. Poult Sci. 1989 Mar;68(3):344-51.

12. Renema RA, Robinson FE. Defining normal: comparison of feed restriction and full feeding of female broiler breeders. Worlds Poult Sci J. 2004 Dec;60(4):508-22.

13. Lees JJ, Lindholm C, Batakis P, Busscher M, Altimiras J. The physiological and neuroendocrine correlates of hunger in the Red Junglefowl (Gallus gallus). Sci Rep. 2017 Dec;7(1):17984.

14. Desert C, Baéza E, Aite M, Boutin M, Le Cam A, Montfort J, et al. Multi-tissue transcriptomic study reveals the main role of liver in the chicken adaptive response to a switch in dietary energy source through the transcriptional regulation of lipogenesis. BMC Genomics. 2018 Dec;19(1):187.

15. de Beer M, Rosebrough RW, Russell BA, Poch SM, Richards MP, Coon CN. An Examination of the Role of Feeding Regimens in Regulating Metabolism During the Broiler Breeder Grower Period. 1. Hepatic Lipid Metabolism. Poult Sci. 2007 Aug 1;86(8):1726-38.

16. Désert C, Duclos MJ, Blavy P, Lecerf F, Moreews F, Klopp C, et al. Transcriptome profiling of the feeding-to-fasting transition in chicken liver. BMC Genomics. 2008 Dec 17;9:611.

17. Kouda K, Nakamura H, Kohno H, Ha-Kawa SK, Tokunaga R, Sawada S. Dietary restriction: effects of short-term fasting on protein uptake and cell death/proliferation in the rat liver. Mech Ageing Dev. 2004 May;125(5):375-80. 
18. Manzanero S, Erion JR, Santro T, Steyn FJ, Chen C, Arumugam TV, et al. Intermittent Fasting Attenuates Increases in Neurogenesis after Ischemia and Reperfusion and Improves Recovery. J Cereb Blood Flow Metab. 2014 May;34(5):897-905.

19. Seroussi E, Cinnamon Y, Yosefi S, Genin O, Smith JG, Rafati N, et al. Identification of the Long-Sought Leptin in Chicken and Duck: Expression Pattern of the Highly GC-Rich Avian leptin Fits an Autocrine/Paracrine Rather Than Endocrine Function. Endocrinology. 2016 Feb;157(2):737-51.

20. Liao BM, Raddatz K, Zhong L, Parker BL, Raftery MJ, Schmitz-Peiffer C. Proteomic analysis of livers from fat-fed mice deficient in either PKCס or PKCE identifies Htatip2 as a regulator of lipid metabolism. PROTEOMICS. 2014 Nov;14(21-22):2578-87.

21. Chen V, Shtivelman E. CC3/TIP30 regulates metabolic adaptation of tumor cells to glucose limitation. Cell Cycle. 2010 Dec 15;9(24):4941-53.

22. Zhao J, Chen J, Lu B, Dong L, Wang H, Bi C, et al. TIP30 Induces Apoptosis under Oxidative Stress through Stabilization of p53 Messenger RNA in Human Hepatocellular Carcinoma. Cancer Res. 2008 Jun 1;68(11):4133-41.

23. Fukuda S, Pelus LM. Survivin, a cancer target with an emerging role in normal adult tissues. Mol Cancer Ther. 2006 May;5(5):1087-98.

24. Bracht T, Hagemann S, Loscha M, Megger DA, Padden J, Eisenacher M, et al. Proteome Analysis of a Hepatocyte-Specific BIRC5 (Survivin)-Knockout Mouse Model during Liver Regeneration. J Proteome Res. 2014 Jun 6;13(6):2771-82.

25. Gasnereau I, Boissan M, Margall-Ducos G, Couchy G, Wendum D, Bourgain-Guglielmetti F, et al. KIF20A mRNA and Its Product MKIp2 Are Increased During Hepatocyte Proliferation and Hepatocarcinogenesis. Am J Pathol. 2012 Jan;180(1):131-40.

26. Brown DT, Wellman SE, Sittman DB. Changes in the levels of three different classes of histone mRNA during murine erythroleukemia cell differentiation. Mol Cell Biol. 1985 Nov;5(11):2879-86.

27. Brocato J, Chen D, Liu J, Fang L, Jin C, Costa M. A Potential New Mechanism of Arsenic Carcinogenesis: Depletion of Stem-Loop Binding Protein and Increase in Polyadenylated Canonical Histone H3.1 mRNA. Biol Trace Elem Res. 2015 Jul;166(1):72-81.

28. Okazaki N. Protocadherin LKC, a new candidate for a tumor suppressor of colon and liver cancers, its association with contact inhibition of cell proliferation. Carcinogenesis. 2002 Jul 1;23(7):1139-48.

29. Xia Z, Huang M, Zhu Q, Li Y, Ma Q, Wang Y, et al. Cadherin Related Family Member 2 Acts As A Tumor Suppressor By Inactivating AKT In Human Hepatocellular Carcinoma. J Cancer. 2019;10(4):864-73.

30. Piotrowska K, Tarnowski M, Zgutka K, Pawlik A. Gender Differences in Response to Prolonged EveryOther-Day Feeding on the Proliferation and Apoptosis of Hepatocytes in Mice. Nutrients. 2016 Mar $19 ; 8(3): 176$.

31. Schneller M, André S, Cihak J, Kaltner H, Merkle H, Janrademaker G, et al. Differential Binding of Two Chicken $\beta$-Galactoside-Specific Lectins to Homologous Lymphocyte Subpopulations and Evidence for Inhibitor Activity of the Dimeric Lectin on Stimulated T Cells. Cell Immunol. 1995 Nov;166(1):3543. 
32. Rabinovich G. Role of galectins in inflammatory and immunomodulatory processes. Biochim Biophys Acta BBA - Gen Subj. 2002 Sep 19;1572(2-3):274-84.

33. Osburn W, Kensler T. Nrf2 signaling: An adaptive response pathway for protection against environmental toxic insults. Mutat Res Mutat Res. 2008 Jul;659(1-2):31-9.

34. Chausse B, Vieira-Lara MA, Sanchez AB, Medeiros MHG, Kowaltowski AJ. Intermittent Fasting Results in Tissue-Specific Changes in Bioenergetics and Redox State. Gueven N, editor. PLOS ONE. 2015 Mar 6;10(3):e0120413.

35. Chen XY, Li R, Geng ZY. Cold stress initiates the Nrf2/UGT1A1/L-FABP signaling pathway in chickens. Poult Sci. 2015 Nov 1;94(11):2597-603.

36. Tomažin U, Frankič Korošec T, Keber R, Rezar V, Horvat S, Salobir J. Oxidative stress response in liver of broiler chickens supplemented with n-3 PUFA-rich linseed oil. Slov Vet Res. 2014 Jan;51(3):10512.

37. Zheng X, O'Connell CM, Zhong W, Poston TB, Wiesenfeld HC, Hillier SL, et al. Gene Expression Signatures Can Aid Diagnosis of Sexually Transmitted Infection-Induced Endometritis in Women. Front Cell Infect Microbiol. 2018 Sep 20;8:307.

38. Dechassa ML, Tryndyak V, de Conti A, Xiao W, Beland FA, Pogribny IP. Identification of chromatinaccessible domains in non-alcoholic steatohepatitis-derived hepatocellular carcinoma. Mol Carcinog. 2018 Aug;57(8):978-87.

39. Van den Bossche L, van Steenbeek FG, Weber MF, Spee B, Penning LC, van Sluijs FJ, et al. Genomewide based model predicting recovery from portosystemic shunting after liver shunt attenuation in dogs. J Vet Intern Med. 2018 Jul;32(4):1343-52.

40. Bugge A, Feng D, Everett LJ, Briggs ER, Mullican SE, Wang F, et al. Rev-erb and Rev-erb coordinately protect the circadian clock and normal metabolic function. Genes Dev. 2012 Apr 1;26(7):657-67.

41. Cho H, Zhao X, Hatori M, Yu RT, Barish GD, Lam MT, et al. Regulation of circadian behaviour and metabolism by REV-ERB-a and REV-ERB- $\beta$. Nature. 2012 May;485(7396):123-7.

42. Yu M, Li W, Wang Q, Wang Y, Lu F. Circadian regulator NR1D2 regulates glioblastoma cell proliferation and motility. Oncogene. 2018 Aug;37(35):4838-53.

43. Schütz KE, Forkman B, Jensen P. Domestication effects on foraging strategy, social behaviour and different fear responses: a comparison between the red junglefowl (Gallus gallus) and a modern layer strain. Appl Anim Behav Sci. 2001 Sep;74(1):1-14.

\section{Figures}




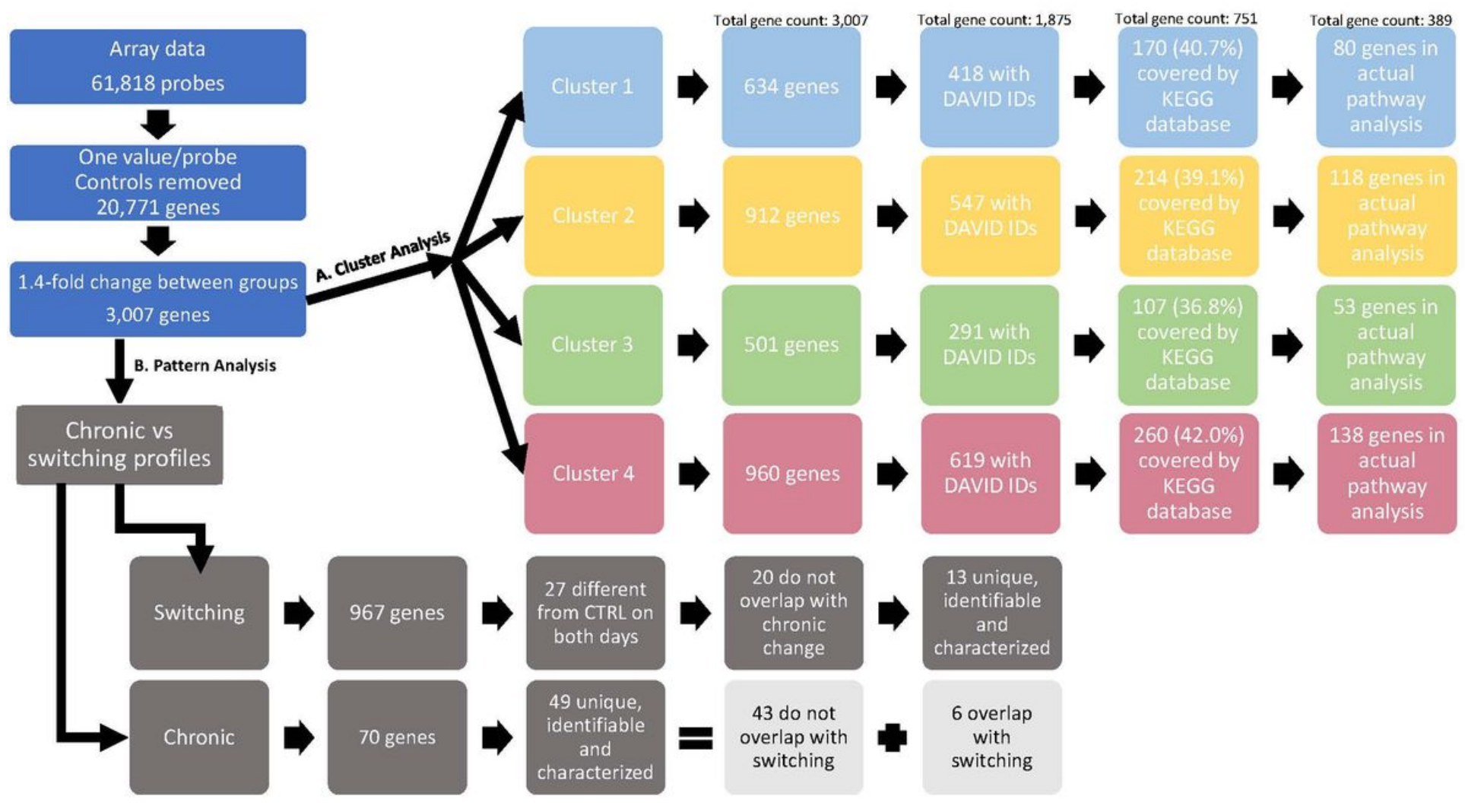

\section{Figure 1}

Flow of data in the analysis. The microarray contained 61,818 probes corresponding to 20,771 genes. A total of 3,007 of these met the criteria of at least 1.4-fold change between groups and were subjected to A) cluster and KEGG pathway analyses, as well as B) a pattern analysis looking for genes showing switching and chronically changed (compared to $\mathrm{AL}$ ) expression patterns. 


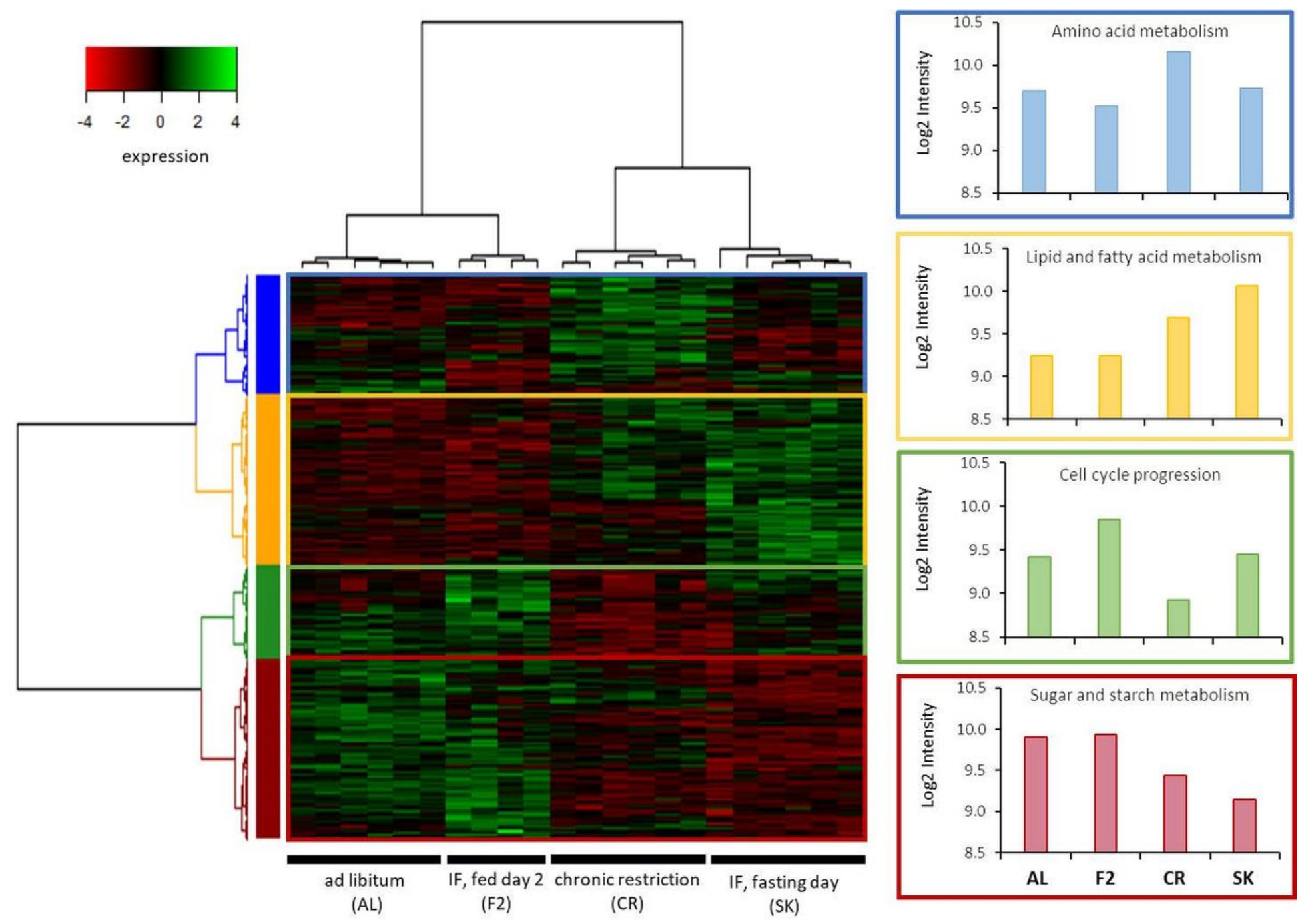

Figure 2

Heatmap of microarray expression data with clusters. The data was clustered into four groups, successfully identifying the four feeding conditions (columns). Overall expression of the four gene clusters (rows) are shown in color-coded panels on the right. Headings for the four clusters have been chosen to represent most of the pathway terms as presented in detail in Fig 4. 

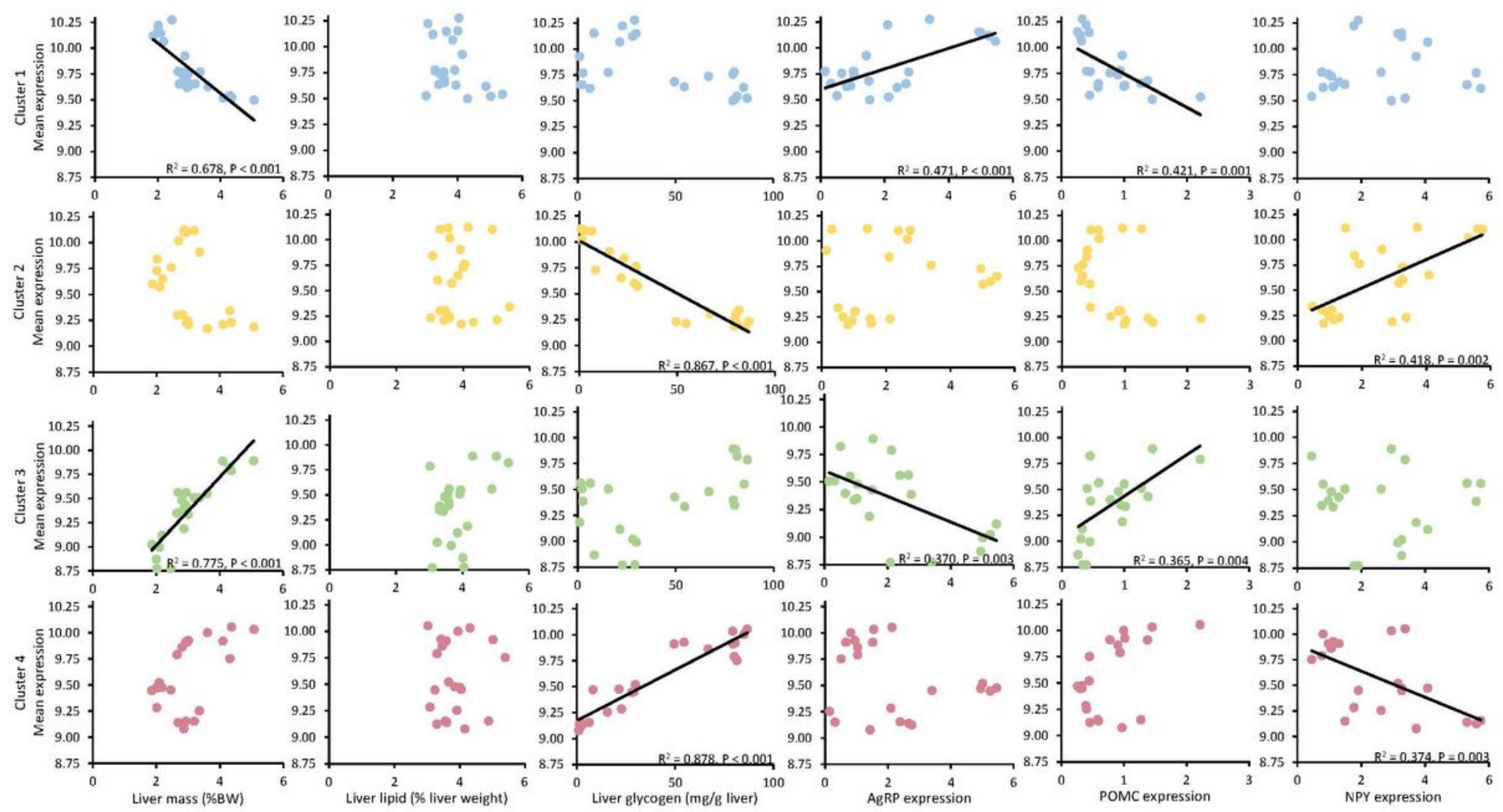

Figure 3

Correlation of mean cluster expression with other measured traits. The mean expression of each clustered underwent correlation analysis with each of seven physiological traits (body mass, absolute liver mass, relative liver mass, total liver lipids, liver lipid concentration, total liver glycogen, liver glycogen concentration) and the expression of three appetite-regulated genes in the arcuate nucleus. Correlations were considered significant at $\mathrm{P}<0.005$ (Bonferroni-corrected from $\mathrm{P}<0.05$ ). 

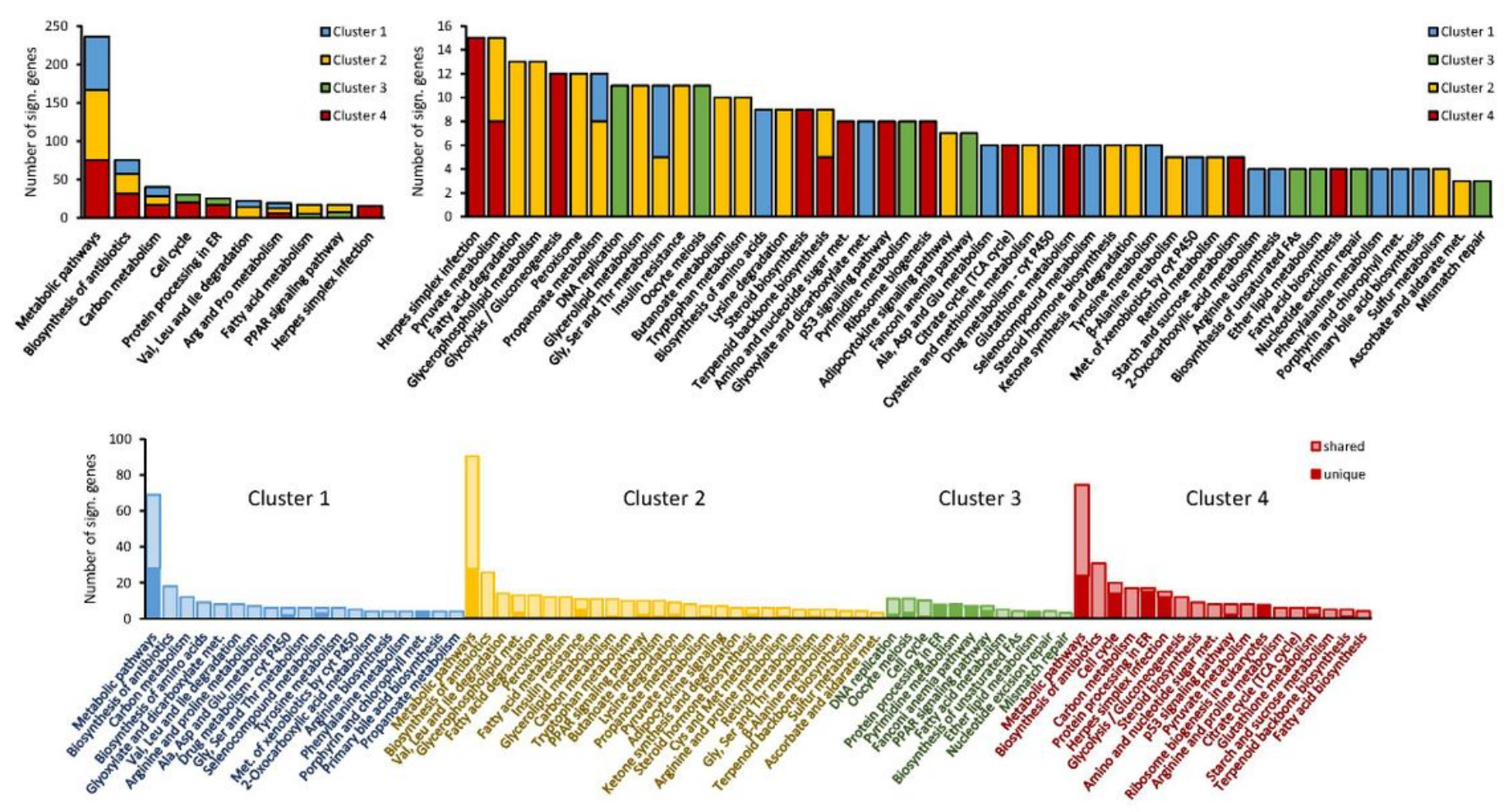

\section{Figure 4}

Results from the KEGG pathway analysis. Top row shows all the pathway terms in order of number of genes (the term "Herpes simplex infection" occurs in both graphs for scale) color-coded for the clusters which they appeared in. Bottom row shows each cluster separately divided into shared (ie. occurring in several of the pathways within the cluster) and unique (ie. only occurring in that one pathway) genes. 

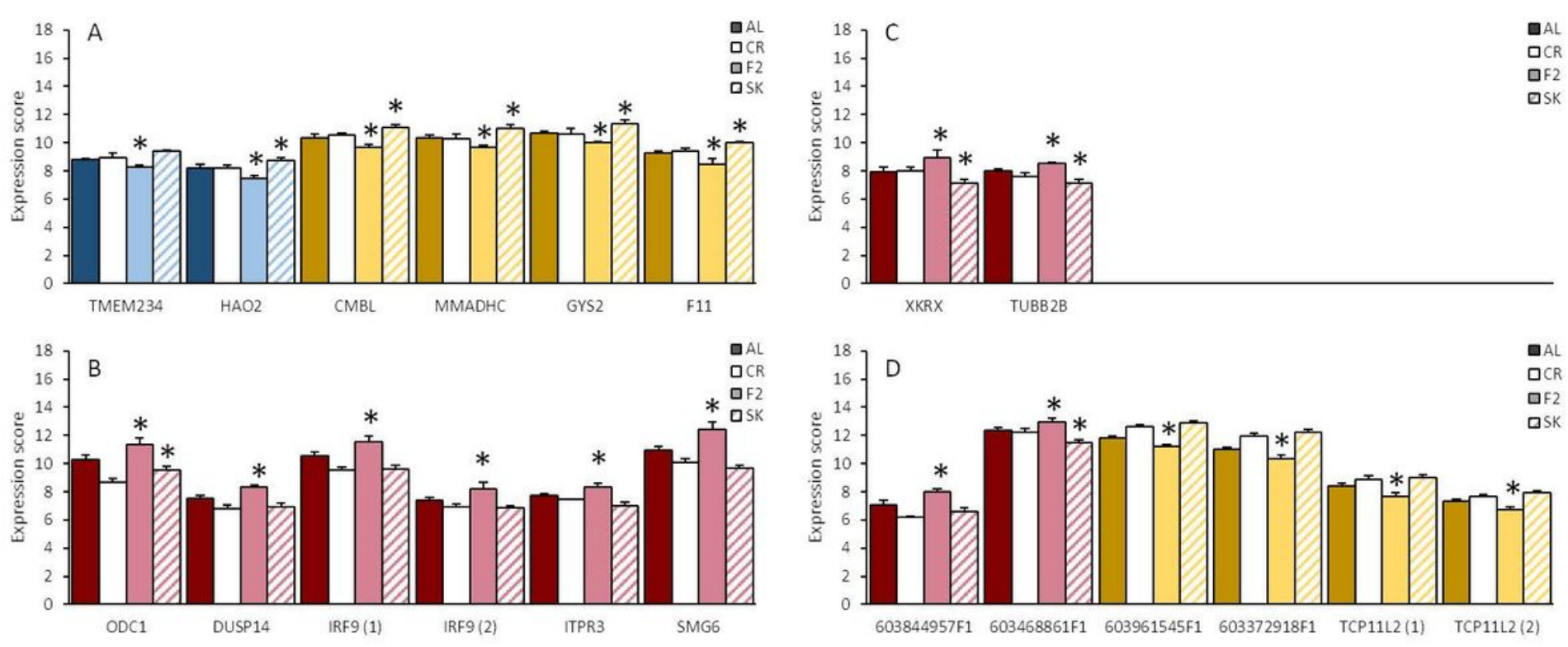

\section{Figure 5}

The expression of 18 genes showing a switching pattern under IF. The gene set is made up of A) six characterized genes that are up-regulated in fasting (SK), B-C) seven characterized genes that are downregulated in fasting and $D$ ) five uncharacterized genes. The expression of these genes is significantly different from $\mathrm{AL}$ on both $\mathrm{F} 2$ and $\mathrm{SK}$ days but in different directions, * indicates significant difference from CR. Colors indicate which cluster each gene appeared in in the cluster analysis.

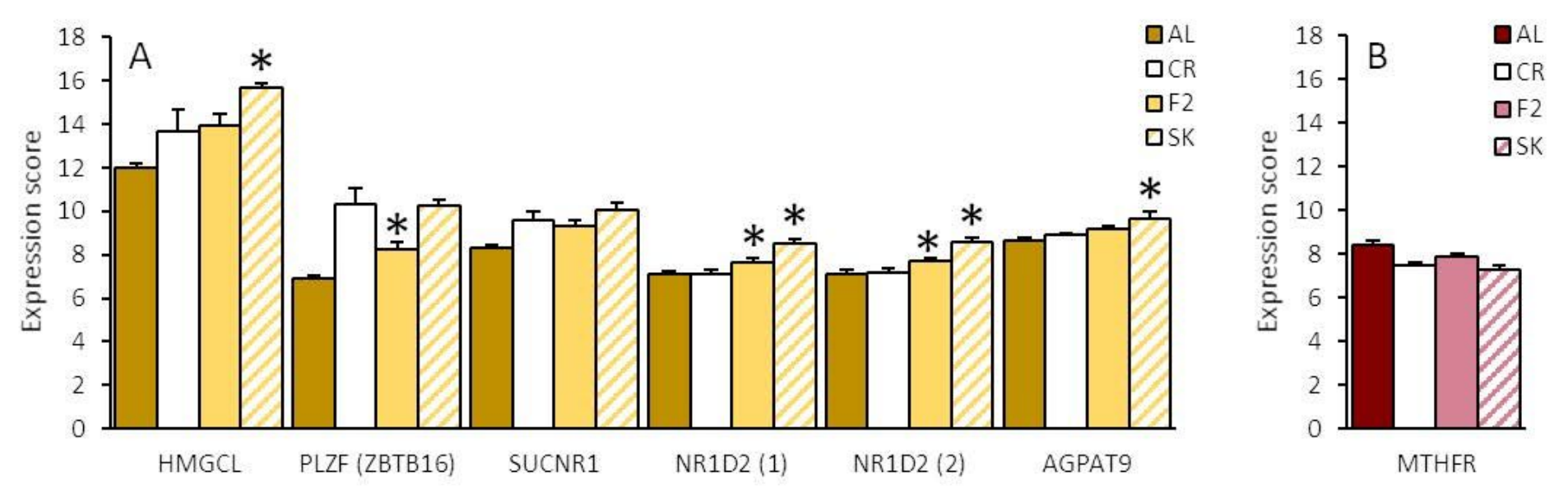

\section{Figure 6}

The expression of six genes showing both chronically changed and switching patterns under IF. This gene set is made up of A) five genes that are up-regulated in fasting and B) one gene that is downregulated in fasting. . The expression of these genes is significantly different from AL on both F2 and SK days in the same direction while F2 and SK expression are also significantly different from each other. * indicates significant difference from CR. Colors indicate which cluster each gene appeared in in the cluster analysis. 

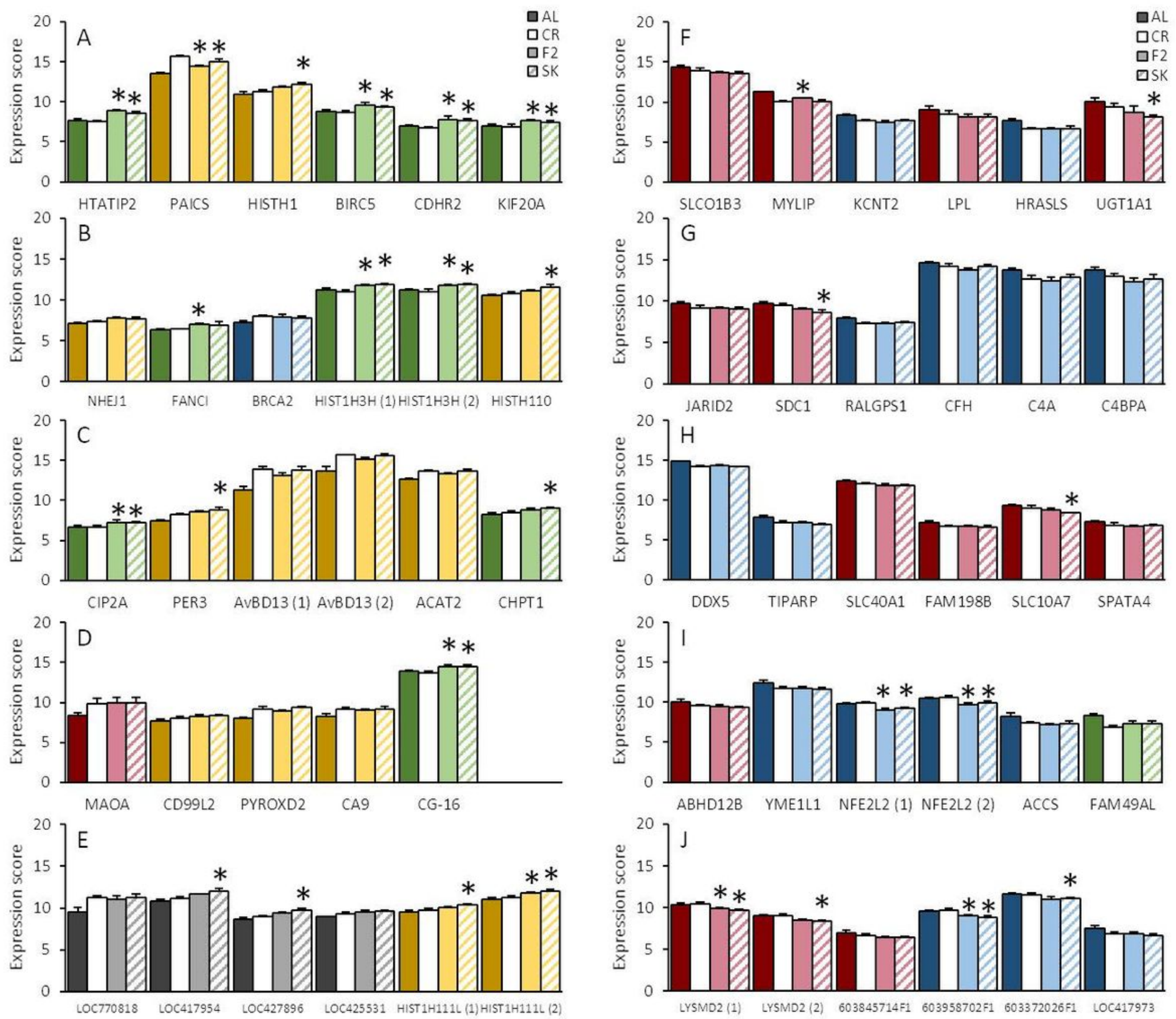

Figure 7

The expression of 54 genes showing a chronic change in gene expression under IF. The gene set is made up of A-D) 21 characterized genes and E) five uncharacterized genes which were chronically up-regulated in IF and F-I) 22 characterized and J) six uncharacterized genes which were down-regulated in IF. The expression of these genes is significantly different from AL on both F2 and SK days, but F2 and SK are not significantly different from each other. * indicates significant difference from CR. Colors indicate which cluster each gene appeared in in the cluster analysis. 

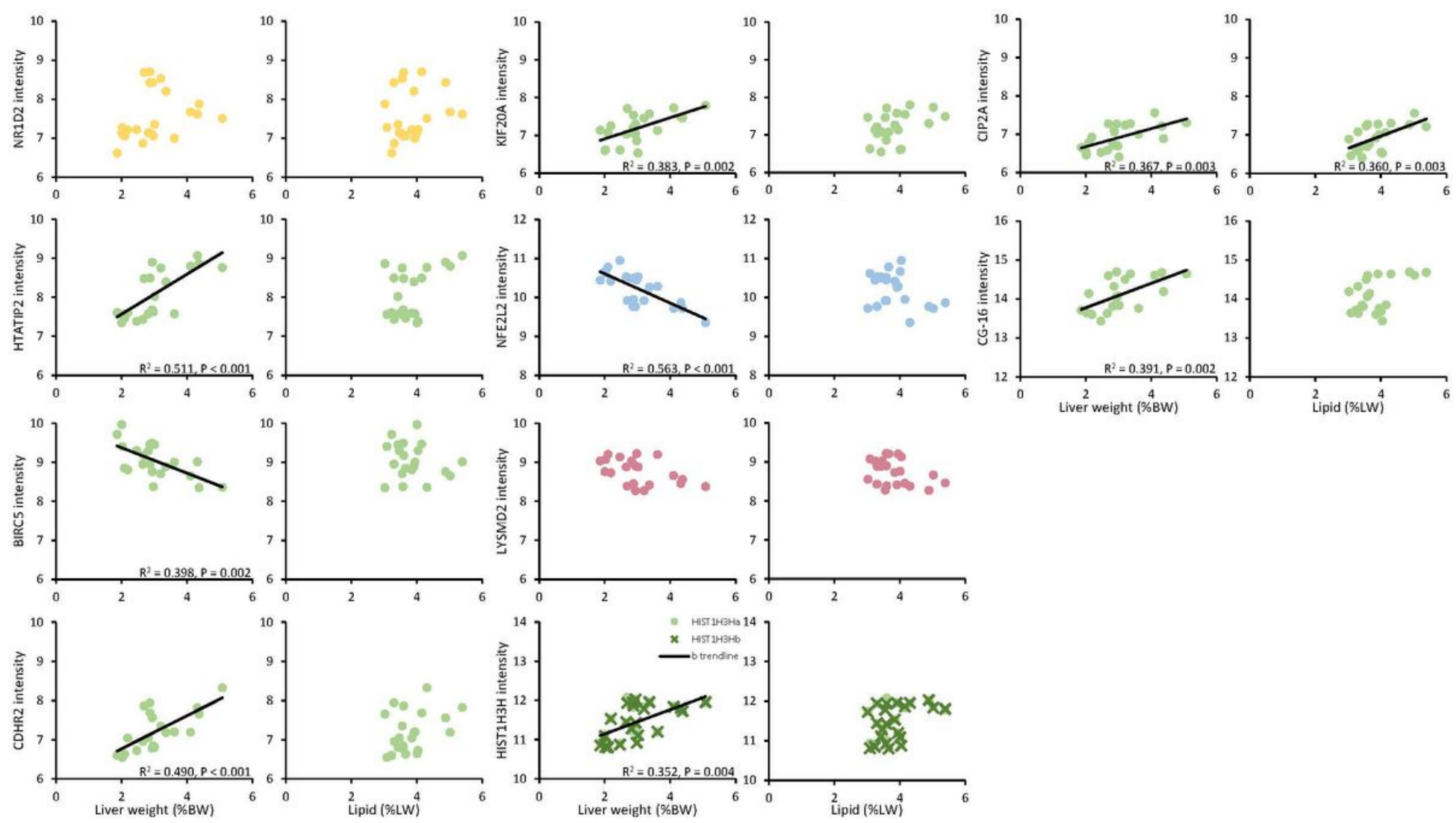

\section{Figure 8}

Correlation of the ten highlighted genes compared to relative liver weight and hepatic lipid concentration. These genes show chronically changed gene expression levels compared to both $A L$ and $C R$. As for the cluster correlations, they were compared against seven physiological traits and the gene expression of three arcuate nucleus appetite-related genes, but only the correlations shown with a trendline here showed a significant correlation at $\mathrm{P}<0.005$ (Bonferroni-corrected from $\mathrm{P}<0.05$ ). Colors indicate which cluster each gene appeared in in the cluster analysis.

\section{Supplementary Files}

This is a list of supplementary files associated with this preprint. Click to download.

- Additional1.eps

- Additional2.eps

- ARRIVEChecklistfilled.pdf 